\title{
Persistent left superior vena cava with absent right superior vena cava in a patient with a secundum atrial septal defect
}

\begin{abstract}
We present a case report of an 11 year old patient, with SitusSolitus, normal segmental anatomy, persistent Left Superior Vena Cava (LSVC) draining into the coronary sinus and absent Right Superior Vena Cava (RSVC). The peculiarity of this presentation was the combination of a LSVC with a secundum Type Atrial Septal Defect (ASD), instead of the more common association to sinus venosus type ASD. From this case, we learned that surgical team's professional experience on rare cardiological pathogenic conditions is the key for survival of patients.
\end{abstract}

Keywords: superior vena cava, LSVC, embryologic, pulmonary veins, CT, venography, MRI
Volume 12 Issue 6 - 2019

\author{
Renzo Otoniel Cifuentes, ${ }^{1,2}$ Clauden Louis, ${ }^{2}$ \\ Maria Virginia Perez, ${ }^{3}$ Boris Barreno, ${ }^{4}$ \\ Mark Gelatt, ${ }^{5}$ David Maldonado, ${ }^{4}$ Marcelo \\ Cardarelli, ${ }^{1,6}$ \\ 'Novick Cardiac Alliance, University of Guayaquil, Ecuador \\ ${ }^{2}$ El CieloFoundations for Children, Ecuador \\ ${ }^{3}$ Department of Cardiothoracic Surgery, University of \\ Rochester Medical Center, USA \\ ${ }^{4}$ Department of Pediatric Cardiology,Hospital Francisco Icaza \\ Bustamante, Ecuador \\ ${ }^{5}$ Department of Pediatric Cardiology, Childrens Mercy hospital, \\ USA \\ ${ }^{6}$ Cardiac Surgery Department, Inova Fairfax, USA
} 56I-6I8-8859, Email rocifuentesmd@gmail.com

Received: November 28, 2019 | Published: December 10, 2019
Abbreviations: RSVC, right superior vena cava; ASD, atrial septal defect; LSVC, left superior vena cava

\section{Case report}

A Casual finding of a murmur in an otherwise asymptomatic 11year old patient, induce further studies. The echocardiography exhibited a secundum ASD and a persistent LSVC draining into a dilated coronary sinus. It was attributed to a poor ultrasound window the inability to visualize a RSVC. The patient was scheduled for surgical repair of the ASD. The particular anatomy of the defect required surgical planing, that included LSVC cannulation through the coronary sinus once the right atrium had been exposed. The team came across an additional challenge in terms of cannulation with the absence of the RSVC. After extensive dissection of the left homolateral pericardium and caudal traction of the left atrial appendage, direct cannulation of the LSVC was accomplished; nonetheless this lead to arrhythmias, modifying even further the patient management plan. Upon successful completion of the repair, the patient remained hemodynamically stable, recovered and was discharged after 4days without sequelae.

\section{Discussion}

A persistent LSVC is one of the most common anomalies of the thoracic venous system. Around $0.3-0.5 \%$ of the general population have this anatomic variant; its presentation increases by 10 folds in patients with congenital heart disease; it most commonly associates with atrial septal defects sinus venosus type. Ninety percent of the cases have a normal right sided SVC; only ten percent are isolated with absent or atretic RSVC(Figures 1-2).

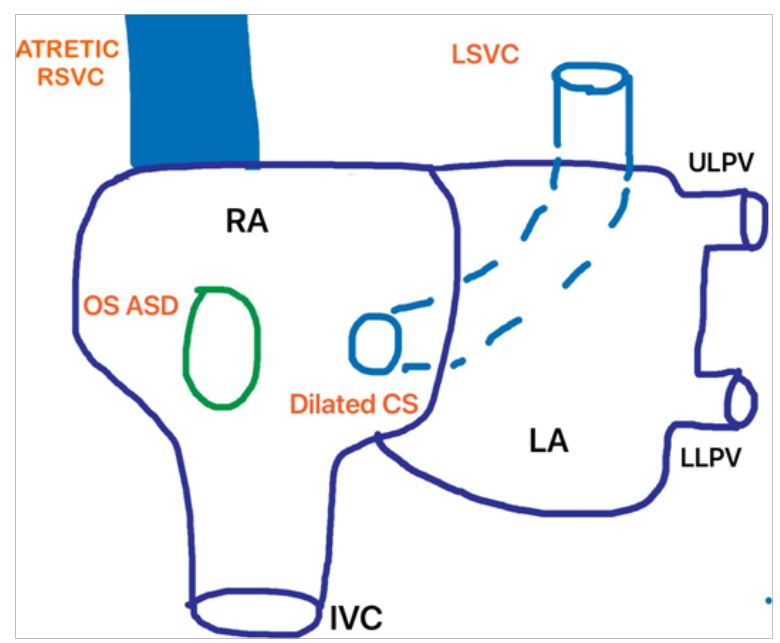

Figure I Diagram of the absent RSVC and persistence of the LSVC draining into a dilated coronary sinus and secundum type ASD.

\section{Embryologic consideration}

The systemic venous return of the heart is formed by the cardinal veins, that drain the blood from the cephalic and caudal portions of the embryo. The anterior and posterior cardinal veins join on each side forming the right and left common cardinal veins. 
Approximately around the 8th week of gestation the development of the innominate vein connects the left and right cardinal veins. The SVC will form from the caudal portion of the right anterior cephalic vein below the transverse anastomosis. On the opposite side this segment will undergo regression, leaving the left brachiocephalic vein draining through the innominate into the the RSV. ${ }^{1}$

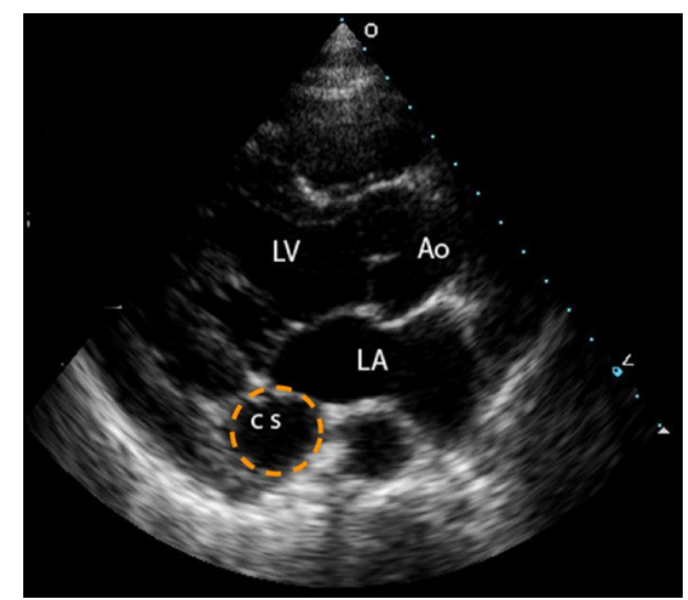

Figure 2 Echo cardiogram: parastern all on gax is view, observing dilated coronary sinus(CS), Aorta(AO), Left Atrium(LA), Left Ventricle(LV).

Two anomalies can arise based on variations in the development of the cardinal veins. Failure in the development of the left brachiocephalic vein leads to a Double SVC. On the other hand PLSVC with absence of the RSVC; is due to persistence of the left anterior cardinal vein, and obliteration of the common cardinal vein and right anterior cardinal vein. The LSVC will drain into the right atrium via the coronary sinus, runing posteriorly one the left side of the heart, between the left atrial appendage and the left pulmonary veins, until it reaches the right atrium.

The diagnosis can be made by Echocardiogram: transthoracic (TTE) or transesophageal (TEE). TEE has an increased sensitivity and also facilitates the visualization of associated anomalies. On TTE A three vessel view on the echocardiogram will contribute to the diagnosis, going from left to right the vessels observed are the ductusarteriosus, aortic arch and RSVC, if the latter is absent then the LSVC will be seen on the left of the arterial duct. Confirmation is then made via long axis view showing the LSVC draining into a dilated coronary sinus. ${ }^{2,3}$ Confirmation can be achieved via CT, venography, or MRI.

\section{Conclusion}

Anatomic considerations after accurate diagnosis are fundamental when working up congenital heart defects. Anatomic associations may be common but not exclusive, as seen with this patient. From this case, we learned that surgical team's professional experience on rare cardiological pathogenic conditions is the key for survival of patients.

\section{Acknowledgments}

None.

\section{Conflicts of interest}

Author declares that there are no conflicts of interest.

\section{Funding}

None.

\section{References}

1. Gologorsky E, Macedo FI, Carvalho EM, et al. Unexpected Persistent Left Superior Vena Cava and Absent Right Superior Vena Cava in Situs Solitus Patient. J Card Surg. 25(1):42-45.

2. Higuchi T, Kuroda K, Iida M, et al. Prenatal diagnosis of persistent left and absent right superior vena cava. J Med Ultrasonics. 2013;40(3):261-263.

3. Saha S, Paoletti D, Robertson M. Persistent left superior vena cava - considerations in fetal, pediatric and adult populations. Australas $J$ Ultrasound Med. 2012;15(2):61-66. 